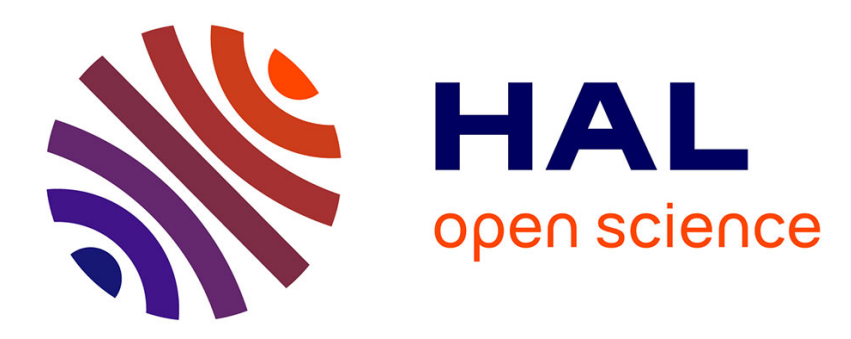

\title{
Limite non visqueuse pour le système de Navier-Stokes dans un espace critique
}

\author{
Taoufik Hmidi, Sahbi Keraani
}

\section{To cite this version:}

Taoufik Hmidi, Sahbi Keraani. Limite non visqueuse pour le système de Navier-Stokes dans un espace critique. Comptes Rendus. Mathématique, 2004, 338 (9), pp.689-692. 10.1016/j.cma.2004.02.013 . hal-00001610

\section{HAL Id: hal-00001610 https://hal.science/hal-00001610}

Submitted on 24 May 2004

HAL is a multi-disciplinary open access archive for the deposit and dissemination of scientific research documents, whether they are published or not. The documents may come from teaching and research institutions in France or abroad, or from public or private research centers.
L'archive ouverte pluridisciplinaire HAL, est destinée au dépôt et à la diffusion de documents scientifiques de niveau recherche, publiés ou non, émanant des établissements d'enseignement et de recherche français ou étrangers, des laboratoires publics ou privés. 


\title{
Limite non visqueuse pour le système de Navier-Stokes dans un espace critique
}

\author{
T. HMidi ${ }^{1}$ et S. Keraani ${ }^{2}$
}

Résumé. Dans un article récent [11], Vishik montre que le système d'Euler bidimensionnel est globalement bien posé dans l'espace de Besov critique $B_{2,1}^{2}$. Nous montrons ici que le système de Navier-Stokes est globalement bien posé dans $B_{2,1}^{2}$, avec des estimations uniformes par rapport à la viscosité. Nous prouvons également un résultat global de limite non visqueuse. Le taux de convergence dans $L^{2}$ est de l'ordre $\nu$.

Abstract. In a recent paper [11], Vishik proved the global well-posedness of the two-dimensional Euler equation in the critical Besov space $B_{2,1}^{2}$. In the present paper we prove that Navier-Stokes system is globally well-posed in $B_{2,1}^{2}$, with uniform estimates on the viscosity. We prove also a global result of inviscid limit. The convergence rate in $L^{2}$ is of order $\nu$.

keywords. navier-Stokes equations; Incompressible inviscid fluids, vorticity flows; viscous-inviscid interaction.

Classification MSC. 76D05, 76C05, 76D09.

\section{Introduction}

L'écoulement d'un fluide visqueux incompressible évoluant dans l'espace tout entier $\mathbb{R}^{d}$ est décrit par le système

$$
\left(N S_{\nu}\right)\left\{\begin{array}{l}
\partial_{t} v_{\nu}+v_{\nu} \cdot \nabla v_{\nu}-\nu \Delta v_{\nu}=-\nabla p_{\nu} \\
\operatorname{div} v_{\nu}=0 \\
v_{\nu \mid t=0}=v^{0},
\end{array}\right.
$$

\footnotetext{
${ }^{1}$ Ecole Polytechnique. E-mail: hmidi@math.polytechnique.fr

${ }^{2}$ Université Rennes 1. E-mail: keraani@maths.univ-rennes1.fr
} 
où $v_{\nu}(t, x) \in \mathbb{R}^{d}$ désigne le champ de vitesse du fluide et $p(t, x) \in \mathbb{R}$ son champ de pression. Le paramètre $\nu>0$ est le cœefficient de viscosité cinématique. Lorsque il n'y a pas de forces de frottement visqueux $(\nu=0)$ alors le système correspondant porte le nom d'Euler :

$$
(E)\left\{\begin{array}{l}
\partial_{t} v+v \cdot \nabla v=-\nabla p \\
\operatorname{div} v=0 \\
v_{\mid t=0}=v^{0}
\end{array}\right.
$$

La théorie qui traite des problèmes d'existence et d'unicité de solutions pour ces deux systèmes est très abondante mais on va se restreindre seulement à quelques résultats significatifs relatives aux solutions fortes. Il convient d'abord de rappeler le fameux résultat de H. Fujita et T. Kato [7] qui date des années soixante et assure pour le système de Navier-Stokes l'existence d'une unique solution locale dans l'espace de Sobolev $H^{s}, s \geq \frac{d}{2}-1$. En revanche le résultat est global dans les deux cas suivants : soit la donnée est petite devant la viscosité, soit $d=2$. En ce qui concerne le système d'Euler, il s'inscrit dans le cadre des systèmes hyperboliques et comme conséquence on montre qu'il est localement bien posé dans les espaces de Sobolev $H^{s}$ dès que $s>\frac{d}{2}+1$ (voir par exemple [9]). En remplaçant l'espace critique $H^{1+d / 2}$ par $B_{2,1}^{1+d / 2}$, alors on peut assurer un résultat local d'existence d'une unique solution (voir par exemple [6]). Lorsqu'on est en dimension deux d'espace alors la solution d'Euler est globale pour toute donnée surcritique. Ceci est fortement lié surtout au fait que le tourbillon $\omega=\partial_{1} v^{2}-\partial_{2} v^{1}$ satisfait une équation de transport et il en découle alors que sa norme $L^{p}$ est conservée. Il est bon de noter que le tourbillon eulérien peut être explicité à chaque instant comme une composition de la donnée initiale et l'inverse du flot $\psi(t)$. Ce dernier est défini par l'équation intégrale :

$$
\psi(t, x)=x+\int_{0}^{t} v(\tau, \psi(\tau, x)) d \tau .
$$

C'est à l'aide de cette représentation que Vishik a établi une estimation logarithmique fine reliant le tourbillon et le flot, ce qui lui a permis de prouver que le système d'Euler bidimensionnel possède une unique solution globale lorsque la donnée initiale est dans l'espace de Besov critique $B_{p, 1}^{1+2 / p}$, avec $p \in] 1,+\infty[$. Les cas extrémaux sont exclus à cause de l'opérateur de Riesz qui n'opère pas continûment dans les espaces de Lebesgue correspondants. Lorsque la donnée initiale est prise dans un espace $B_{p, 1}^{s}$, avec $s<1+2 / p$, alors 
le champ de vitesse n'est pas nécessairement lipschitzien et par conséquent un phénomèmene de dégradation de la régularité au cours du temps est mis en évidence (voir [1]). Concernant la limite non visqueuse, on peut établir que si la donnée initiale est dans un espace surcritique et si la solution d'Euler vit jusqu'à l'instant $T$ alors la solution de Navier-Stokes l'est aussi. De plus, elle converge fortement vers la solution d'Euler sur le même intervalle de temps. Le taux de convergence dans l'espace $L^{2}$ est de l'ordre $\nu$. Ce même résultat persiste encore dans les espaces critiques $B_{p, 1}^{1+d / p}$, cependant il n'est établi que sur un petit intervalle de temps, même en dimension deux.

Dans le présent travail nous allons montrer qu'en dimension deux le système de Navier-Stokes est globalement bien posé dans l'espace critique $B_{2,1}^{2}$, avec un contrôle uniforme par rapport à la viscosité. Nous prouvons également un résultat de limite non visqueuse. La démonstration est fondée sur une estimation uniforme par rapport à la viscosité de la quantité $\left\|v_{\nu}\right\|_{L_{t}^{1} \operatorname{Lip}\left(\mathbb{R}^{2}\right)}$, et l'on utilise à cette fin un effet régularisant de l'équation de transport-diffusion relative à un champ de vecteurs lipschitzien et régissant le tourbillon visqueux $\omega_{\nu}=\partial_{1} v_{\nu}^{2}-\partial_{2} v_{\nu}^{1}$ :

$$
\left(T D_{\nu}\right) \quad \partial_{t} \omega_{\nu}+v_{\nu} \cdot \nabla \omega_{\nu}-\nu \Delta \omega_{\nu}=0 .
$$

Voici notre principal théorème.

Théorème 1. Soit $v^{0}$ un champ de vecteurs de $\mathbb{R}^{2}$ à divergence nulle et appartenant à $B_{2,1}^{2}$. Alors le système $\left(N S_{\nu}\right)$ possède une unique solution globale dans $L_{\text {loc }}^{\infty}\left(\mathbb{R}_{+} ; B_{2,1}^{2}\right)$ satisfaisant l'estimation uniforme en $\nu$

$$
\left\|v_{\nu}(t)\right\|_{B_{2,1}^{2}} \leq C\left\|v^{0}\right\|_{B_{2,1}^{2}} \exp \left(e^{\exp C t\left\|v^{0}\right\|_{B_{2,1}^{2}}}\right) .
$$

De plus, la solution de $\left(N S_{\nu}\right)$ converge vers celle d'Euler, quand la viscosité tend vers zéro, dans tous les espaces $B_{2,1}^{2-s}$, pour tout $s>0$ :

$$
\left\|v_{\nu}(t)-v(t)\right\|_{L^{2}} \leq C(\nu t)\left\|v^{0}\right\|_{B_{2,1}^{2}} e^{\exp C t\left\|v^{0}\right\|_{B_{2,1}^{2}}} .
$$

Le plan de l'article est structuré comme suit : la première section est un rappel de la théorie de Littlewood-Paley suivi de l'énoncé de quelques lemmes techniques bien connus. Nous démontrons dans la section d'après l'estimation (1) qui repose sur le résultat de Vishik dans le cas d'Euler. Nous décrirons dans le paragraphe qui suit l'effet régularisant développé dans l'équation $\left(T D_{\nu}\right)$. Par contre la fin de l'article est consacrée à la preuve du résultat de propagation de la régularité, uniformément en $\nu$. 


\section{Outils de base}

Nous allons définir dans cette partie les opérateurs de localisation en fréquences. Nous donnons également la définition des espaces de Besov $B_{p, 1}^{s}$ et nous achevons ce paragraphe par quelques lemmes fort utiles pour la preuve des résultats énoncés.

Proposition 1. Il existe deux fonctions radiales $\chi$ et $\phi$ appartenant respectivement aux espaces $\mathcal{D}\left(\mathbb{R}^{d}\right)$ et $\mathcal{D}\left(\mathbb{R}^{d} \backslash\{0\}\right)$ telles que,

$$
\begin{gathered}
\chi(\xi)+\sum_{q \geq 0} \varphi\left(2^{-q} \xi\right)=1, \quad \frac{1}{3} \leq \chi^{2}(\xi)+\sum_{q \geq 0} \varphi^{2}\left(2^{-q} \xi\right) \leq 1, \\
|p-q| \geq 2 \Rightarrow \operatorname{supp} \varphi\left(2^{-p} \cdot\right) \cap \operatorname{supp} \varphi\left(2^{-q} \cdot\right)=\varnothing, \\
q \geq 1 \Rightarrow \operatorname{supp} \chi \cap \operatorname{supp} \varphi\left(2^{-q}\right)=\varnothing .
\end{gathered}
$$

On pose alors pour toute distribution tempérée $v$

$$
\Delta_{-1} v=\chi(D) v ; \quad \forall q \in \mathbb{N}, \quad \Delta_{q} v=\varphi\left(2^{-q} D\right) v \quad \text { et } \quad S_{q} v=\sum_{-1 \leq p \leq q-1} \Delta_{p} v .
$$

Le calcul paradifférentiel introduit par J.-M. Bony dans [2] est basé sur la décomposition, dite de Bony, qui reconnaît dans un produit $u v$ trois parties: deux termes de paraproduits correspondant à une domination fréquencielle de l'une par rapport à l'autre et un terme de reste où les fréquences sont de même taille. Plus précisément, nous avons la définition suivante

Définition 1. On appelle paraproduit de $v$ par $u$ et on note $T_{u} v$ l'opérateur

$$
T_{u} v=\sum_{q} S_{q-1} u \Delta_{q} v
$$

On appelle reste du produit uv et on note $R(u, v)$ l'opérateur bilinéaire symétrique suivant :

$$
R(u, v)=\sum_{\left|q^{\prime}-q\right| \leq 1} \Delta_{q} u \Delta_{q^{\prime}} v
$$

Ainsi le produit uv s'écrit formellement

$$
u v=T_{u} v+T_{v} u+R(u, v) .
$$


Nous définissons les espaces de Besov $B_{p, 1}^{s}$, avec $p \in[1,+\infty]$ et $s \in \mathbb{R}$ comme étant l'ensemble des distributions tempérées $u$ vérifiant

$$
\|u\|_{B_{p, 1}^{s}} \stackrel{\text { déf }}{=} \sum_{q=-1}^{+\infty} 2^{q s}\left\|\Delta_{q} u\right\|_{L^{p}}<+\infty .
$$

La chaîne d'espaces $\left(B_{p, 1}^{s}\right)_{s}$ est décroissante. Nous avons de plus l'inclusion de Sobolev :

$$
B_{p, 1}^{2 / p} \hookrightarrow L^{\infty}
$$

Nous aurons également besoin de la description suivante de l'effet régularisant du semi-groupe de la chaleur:

Lemme 1. Soit $\mathcal{C}$ une couronne. Il existe deux constantes positives $c$ et $C$ telles que, pour tout couple $(t, \lambda)$ de réels positifs, pour tout $p \in[1,+\infty]$ et pour tout $a \in L^{p}$, on aura l'implication:

$$
\text { Supp } \widehat{a} \subset \lambda \mathcal{C} \Rightarrow\left\|e^{t \Delta} a\right\|_{L^{p}} \leq C e^{-c t \lambda^{2}}\|a\|_{L^{p}}
$$

Nous ferons usage du lemme de Bernstein qui décrit le coût d'une différentiation d'une distribution. Plus précisément nous avons

Lemme 2. (BERNSTEIN) Soit $\left(r_{1}, r_{2}\right)$ un couple de réels strictement positifs tels que $r_{1}<r_{2}$. Il existe une constante $C$ telle que, pour tout entier $k$, pour tout couple $(a, b)$ tel que $1 \leq a \leq b$ et pour toute fonction $u \in L^{a}\left(\mathbb{R}^{d}\right)$, on ait

$$
\begin{aligned}
\operatorname{supp} \widehat{u} \in B\left(0, \lambda r_{1}\right) & \Rightarrow \sup _{|\alpha|=k}\left\|\partial^{\alpha} u\right\|_{L^{b}} \leq C^{k} \lambda^{k+d\left(\frac{1}{a}-\frac{1}{b}\right)}\|u\|_{L^{a}}, \\
\operatorname{supp} \widehat{u} \in \mathcal{C}\left(0, \lambda r_{1}, \lambda r_{2}\right) & \Rightarrow C^{-k} \lambda^{k}\|u\|_{L^{a}} \leq \sup _{|\alpha|=k}\left\|\partial^{\alpha} u\right\|_{L^{a}} \leq C^{k} \lambda^{k}\|u\|_{L^{a}} .
\end{aligned}
$$

\section{Limite non visqueuse}

Ce paragraphe est consacré à la preuve de l'estimation (1). Elle se démontre via une estimation d'énergie et fait appel aux estimations suivantes, prouvées dans [11]:

$$
\|v(t)\|_{B_{2,1}^{2}} \leq C\left\|v^{0}\right\|_{B_{2,1}^{2}} e^{\exp C t\left\|v^{0}\right\|_{B_{2,1}^{2}}} \quad \text { et } \quad\|\nabla v(t)\|_{L^{\infty}} \leq e^{C t\left\|v^{0}\right\|_{B_{2,1}^{2}}}
$$

Posons

$$
W_{\nu}=v_{\nu}-v .
$$


Alors en combinant les équations $\left(N S_{\nu}\right)$ et $(E)$, on trouve que $W_{\nu}$ satisfait l'équation

$$
\left\{\begin{array}{l}
\partial_{t} W_{\nu}+v_{\nu} \cdot \nabla W_{\nu}-\nu \Delta W_{\nu}+\nabla p_{\nu}=\nu \Delta v+W_{\nu} \cdot \nabla v \\
\operatorname{div} W_{\nu}=0 \\
W_{\nu \mid t=0}=0 .
\end{array}\right.
$$

En faisant le produit scalaire $L^{2}$ de cette équation avec $W_{\nu}$ et en se servant de la contrainte d'incompressibilité, on aboutit à

$$
\frac{1}{2} \frac{d}{d t}\left\|W_{\nu}(t)\right\|_{L^{2}}^{2}+\nu\left\|\nabla W_{\nu}(t)\right\|_{L^{2}}^{2} \leq \nu\|\Delta v\|_{L^{2}}\left\|W_{\nu}\right\|_{L^{2}}+\|\nabla v\|_{L^{\infty}}\left\|W_{\nu}\right\|_{L^{2}}^{2} .
$$

Donc cela entraîne suite à une intégration en temps et une application du lemme de Gronwall,

$$
\left\|W_{\nu}(t)\right\|_{L^{2}} \leq \nu \int_{0}^{t}\|\Delta v(\tau)\|_{L^{2}} d \tau e^{\int_{0}^{t}\|\nabla v(\tau)\|_{L^{\infty} d \tau}}
$$

Il suffit à ce stade d'utiliser les estimations (2) pour déduire (1).

\section{Effet régularisant}

Le but de cette section est de décrire le gain d'une moyenne en temps du tourbillon visqueux. Le point le plus important est d'avoir le gain souhaité avec une croissane linéaire et non pas exponentielle de la norme Lipschitz de la vitesse. Un tel résultat est déjà établi dans [8] mais nous le redémontrons pour la commodité du lecteur.

Proposition 2. Soit $\omega^{0} \in L^{p}$, avec $p \in[1,+\infty]$ et $v_{\nu}$ une solution de $\left(N S_{\nu}\right)$ qui soit lipschitzienne. Alors, pour tout $q \in \mathbb{N}$ on a l'estimation

$$
\nu 2^{2 q} \int_{0}^{t}\left\|\Delta_{q} \omega_{\nu}(\tau)\right\|_{L^{p}} d \tau \leq C\left\|\omega^{0}\right\|_{L^{p}}\left(1+\int_{0}^{t}\left\|\nabla v_{\nu}(\tau)\right\|_{L^{\infty}} d \tau\right) .
$$

Démonstration : La preuve se fait en deux temps : en premier lieu nous démontrons l'effet régularisant sur un petit intervalle de temps qui ne dépend pas de la donnée initiale mais dépendant uniquement du champ de vecteurs $v$. Ensuite, nous procédons à un découpage en temps permettant ainsi d'étendre les estimations à n'importe quel temps arbitrairement positif. 


\section{Estimation Locale}

Nous commençons par localiser en fréquences l'équation $\left(T D_{\nu}\right)$ qui gouverne le tourbillon visqueux, noté $\omega$ (afin d'alléger les notations). Alors, en posant $\omega_{q}=\Delta_{q} \omega$, nous obtenons

$$
\begin{aligned}
\partial_{t} \omega_{q}+S_{q-1} v \cdot \nabla \omega_{q}-\nu \Delta \omega_{q} & =-\left[\Delta_{q}, v \cdot \nabla\right] \omega+\left(S_{q-1} v-v\right) \cdot \nabla \omega_{q}, \\
& \stackrel{\text { déf }}{=} f_{q} .
\end{aligned}
$$

Remarquons d'abord que le spectre fréquenciel de $f_{q}$ est localisé dans une coronne de taille $2^{q}$ et que l'on a aussi, en vertu de la proposition 3 , l'estimation

$$
\left\|f_{q}(t)\right\|_{L^{p}} \leq C\|\nabla v(t)\|_{L^{\infty}}\|\omega(t)\|_{L^{p}} .
$$

Or nous savons que la norme $L^{p}$ est majorée à chaque insatant $t$ par sa valeur initiale. Ainsi, nous en déduisons que

$$
\left\|f_{q}(t)\right\|_{L^{p}} \leq C\|\nabla v(t)\|_{L^{\infty}}\left\|\omega^{0}\right\|_{L^{p}} .
$$

Nous allons maintenant faire le changement de variables lagrangiens dans l'équation (3). Posons

$$
\bar{\omega}_{q}(t, x)=\omega_{q}\left(t, \psi_{q}(t, x)\right) \quad \text { et } \quad \bar{f}_{q}(t, x)=f_{q}\left(t, \psi_{q}(t, x)\right) .
$$

En écrivant $\omega_{q}(t, x)=\bar{\omega}_{q}\left(t, \psi_{q}^{-1}(t, x)\right)$ et en calculant son laplacien à l'aide de la formule de dérivation de fonctions composées, alors, en notant la hessienne de $\bar{\omega}_{q}$ par $\nabla^{2} \bar{\omega}_{q}$, on trouve

$$
\begin{array}{r}
\Delta \omega_{q}(t) \circ \psi_{q}(t, x)=\sum_{i=1}^{d}\left\langle\nabla^{2} \bar{\omega}_{q}(t, x) \cdot\left(\partial^{i} \psi_{q}^{-1}\right)\left(t, \psi_{q}(t, x)\right),\left(\partial^{i} \psi_{q}^{-1}\right)\left(t, \psi_{q}(t, x)\right)\right\rangle \\
+\nabla \bar{\omega}_{q}(t, x) \cdot\left(\Delta \psi_{q}^{-1}\right)\left(t, \psi_{q}(t, x)\right) .
\end{array}
$$

Le symbole $\langle\cdot, \cdot\rangle$ désigne le produit scalaire canonique de $\mathbb{R}^{2}$. Pour le contrôle des dérivées du flot et de son inverse $\psi_{q}^{-1}(t, x)$, nous disposons des estimations classiques

$$
\left\|\nabla \psi_{q}^{\mp 1}(t)\right\|_{L^{\infty}} \leq \exp \left(\int_{0}^{t}\left\|\nabla S_{q-1} v(\tau)\right\|_{L^{\infty}} d \tau\right) .
$$

Dorénavant on adopte les notations suivantes :

$$
V(t)=\int_{0}^{t}\|\nabla v(\tau)\|_{L^{\infty}} d \tau \quad \text { et } \quad V_{q}(t)=\int_{0}^{t}\left\|\nabla S_{q-1} v(\tau)\right\|_{L^{\infty}} d \tau .
$$


Quand on dérive l'équation d'évolution régissant l'inverse du flot par rapport à la variable d'espace alors on peut déduire, grâce à (6), que la fonction $\psi_{q}^{-1}$ satisfait une équation de type

$$
\left(\partial^{i} \psi_{q}^{-1}\right)\left(t, \psi_{q}(t, x)\right)=e_{i}+g_{q}^{i}(t, x)
$$

avec $e_{i}$ le $i^{\text {eme }}$ vecteur canonique de $\mathbb{R}^{2}$. De surcroît, la fonction $g_{q}^{i}$ se majore comme suit :

$$
\left\|g_{q}^{i}(t)\right\|_{L^{\infty}} \leq \exp \left(\int_{0}^{t} \| \nabla S_{q-1} v(\tau) d \tau\right) \int_{0}^{t}\left\|\nabla S_{q-1} v(\tau)\right\|_{L^{\infty}} d \tau .
$$

Comme $S_{q-1}$ envoie uniformément en $q$ l'espace $L^{\infty}$ dans lui-même, alors nous aurons

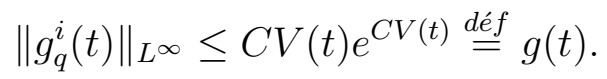

En ce qui concerne l'estimation des dérivées secondes de $\psi_{q}^{-1}(t)$, nous dérivons deux fois l'équation d'évolution de $\psi_{q}^{-1}$ et nous obtenons, suite à une application du lemme de Gronwall, que

$$
\left\|\nabla^{2} \psi_{q}^{-1}(t)\right\|_{L^{\infty}} \leq e^{V_{q}(t)} \int_{0}^{t}\left\|\nabla^{2} S_{q-1} v(\tau)\right\|_{L^{\infty}} d \tau .
$$

Ainsi donc, par le biais de l'inégalité de Bernstein et l'estimation (6), l'inégalité (9) devient

$$
\left\|\nabla^{2} \psi_{q}^{\mp 1}(t)\right\|_{L^{\infty}} \leq C 2^{q} g(t) .
$$

Intéressons-nous maintenant à l'équation satisfaite par $\bar{\omega}_{q}$. Alors un simple calcul utilisant les informations $(3)$, (5) et (7) montre que la fonction $\bar{\omega}_{q}$ vérifie

$$
\begin{aligned}
\left(\partial_{t}-\nu \Delta\right) \bar{\omega}_{q}(t, x) & =\nu \sum_{i=1}^{d}\left\langle\nabla^{2} \bar{\omega}_{q}(t, x) g_{q}^{i}, g_{q}^{i}(t, x)\right\rangle+2 \nu \sum_{i=1}^{d}\left\langle\nabla^{2} \bar{\omega}_{q}(t, x) e_{i}, g_{q}^{i}(t, x)\right\rangle \\
& +\nu \nabla \bar{\omega}_{q}(t, x) \cdot\left(\Delta \psi_{q}^{-1}\right)\left(t, \psi_{q}(t, x)\right)+\bar{f}_{q}(t, x) \stackrel{\text { déf }}{=} \mathcal{R}_{q}(t, x) \cdot(11)
\end{aligned}
$$

Comme la fonction composée $\bar{\omega}_{q}$ n'est pas nécessairement localisée en fréquences, alors nous ne pouvons pas appliquer immédiatement le lemme 1. Donc nous serons amenés à tronquer l'équation (11) via l'opérateur $\Delta_{j}$, avec $j \in \mathbb{N}$. Ainsi la formule de Duhamel montre que la fonction $\Delta_{j} \bar{\omega}_{q}$ vérifie

$$
\Delta_{j} \bar{\omega}_{q}(t, x)=e^{\nu t \Delta} \Delta_{j} \omega_{q}(0)+\int_{0}^{t} e^{\nu(t-\tau) \Delta} \Delta_{j} \mathcal{R}_{q}(\tau, x) d \tau .
$$


Le support de $\widehat{f}_{q}$ est localisé dans une boule de taille $2^{q}$. Donc, il s'ensuit, grâce au lemme de Bernstein et la préservation de la mesure par le flot, que

$$
\begin{aligned}
\left\|\Delta_{j} \bar{f}_{q}(t)\right\|_{L^{p}} & \leq C 2^{-j}\left\|\nabla\left(f_{q} \circ \psi_{q}(t)\right)\right\|_{L^{p}}, \\
& \leq C 2^{q-j}\left\|\nabla \psi_{q}(t)\right\|_{L^{\infty}}\left\|f_{q}(t)\right\|_{L^{p}} .
\end{aligned}
$$

Ainsi les inégalités (4) et (6) permettent d'avoir

$$
\begin{aligned}
\left\|\Delta_{j} \bar{f}_{q}(t)\right\|_{L^{p}} & \leq C 2^{q-j} e^{V_{q}(t)}\left\|\omega^{0}\right\|_{L^{p}}\|\nabla v(t)\|_{L^{\infty}} \\
& \leq C 2^{q-j} e^{C V(t)}\left\|\omega^{0}\right\|_{L^{p}}\|\nabla v(t)\|_{L^{\infty}} .
\end{aligned}
$$

En conséquence, nous déduisons, à l'aide du lemme 1 et des inégalités (8) et (10), que

$$
\begin{aligned}
\left\|\Delta_{j} \bar{\omega}_{q}(t)\right\|_{L^{p}} \leq & C e^{-c \nu t 2^{2 j}}\left\|\Delta_{j} \omega_{q}(0)\right\|_{L^{p}}+ \\
& +C \nu\left(g(t)+g^{2}(t)\right) \int_{0}^{t} e^{-c \nu(t-\tau) 2^{2 j}}\left\|\nabla^{2} \bar{\omega}_{q}(\tau)\right\|_{L^{p}} d \tau+ \\
& +C \nu 2^{q} g(t) \int_{0}^{t} e^{-c \nu(t-\tau) 2^{2 j}}\left\|\nabla \bar{\omega}_{q}(\tau)\right\|_{L^{p}} d \tau+ \\
& +C 2^{q-j} e^{C V(t)}\left\|\omega^{0}\right\|_{L^{p}} \int_{0}^{t} e^{-c \nu(t-\tau) 2^{2 j}}\|\nabla v(\tau)\|_{L^{\infty}} d \tau .
\end{aligned}
$$

Prenons la norme $L^{1}$ en temps dans les deux membres de l'inégalité (13). Alors nous obtenons par le biais de l'inégalité de convolution retardée et la croisssance de $g$,

$$
\begin{aligned}
\left\|\Delta_{j} \bar{\omega}_{q}(t)\right\|_{L^{1}\left([0, t] ; L^{p}\right)} \leq & C\left(\nu 2^{2 j}\right)^{-1}\left\|\Delta_{j} \omega_{q}(0)\right\|_{L^{p}}+ \\
& +C\left(g(t)+g^{2}(t)\right) 2^{-2 j}\left\|\nabla^{2} \bar{\omega}_{q}\right\|_{L^{1}\left([0, t] ; L^{p}\right)} \\
& +C g(t) 2^{q} 2^{-2 j}\left\|\nabla \bar{\omega}_{q}\right\|_{L^{1}\left([0, t] ; L^{p}\right)} \\
& +C g(t)\left\|\omega^{0}\right\|_{L^{p}} 2^{q-j}\left(\nu 2^{2 j}\right)^{-1}
\end{aligned}
$$

En se servant de (6), (10) et du lemme de Bernstein on aboutit, grâce à la conservation de la mesure par le flot, aux estimations

$$
\begin{aligned}
\left\|\nabla \bar{\omega}_{q}(t)\right\|_{L^{p}} & \leq C 2^{q} e^{C V(t)}\left\|\omega_{q}(t)\right\|_{L^{p}} \\
\left\|\nabla^{2} \bar{\omega}_{q}(t)\right\|_{L^{p}} & \leq C 2^{2 q} e^{C V(t)}\left\|\omega_{q}(t)\right\|_{L^{p}}
\end{aligned}
$$


Soit $N_{0}$ un entier que l'on choisira à la fin. Alors en reportant (15) et (16) dans (14) et en sommant sur les entiers $j \geq q-N_{0}$, on trouve

$$
\begin{array}{r}
\nu 2^{2 q} \sum_{j \geq q-N_{0}}\left\|\Delta_{j} \bar{\omega}_{q}(t)\right\|_{L^{1}\left([0, t] ; L^{p}\right)} \leq C\left(\left\|\omega^{0}\right\|_{L^{p}}+2^{2 N_{0}} h(t)\left(\nu 2^{2 q}\right)\left\|\omega_{q}\right\|_{L^{1}\left([0, t] ; L^{p}\right)}\right. \\
\left.+2^{3 N_{0}}\left\|\omega^{0}\right\|_{L^{p}} g(t)\right), \quad(17)
\end{array}
$$

où l'on a posé $h(t)=g(t)+g(t)^{2}$. Faisons remarquer qu'il n'y a pas un cœefficient en $N_{0}$ dans le premier terme du second membre de (17) car $\Delta_{j} \omega_{q}(0)=0$, si $|j-q| \geq 2$. Il faut noter aussi que le calcul que nous venons de développer est valable pour les entiers $q \geq N_{0}$, sinon, l'estimation (17) serait fausse. Concernant le cas des basses fréquences $j \leq q-N_{0}$, on dispose du lemme suivant, démontré par M. Vishik dans [11].

Lemme 3. Soit d un entier supérieur ou égal à 2 . Il existe une constante positive $C$ ne dépendant que de d telle que, pour toute fonction a de la classe de Schwartz et pour tout difféomorphisme $\psi$ de $\mathbb{R}^{d}$ préservant la mesure de Lebesgue, on aura pour tout $p \in[1,+\infty]$ et pour tous les $j, q \geq-1$,

$$
\left\|\Delta_{j}\left(\Delta_{q} a \circ \psi(\cdot)\right)\right\|_{L^{p}} \leq C 2^{-|j-q|}\left\|\nabla \psi^{\alpha(j, q)}\right\|_{L^{\infty}}\left\|\Delta_{q} a\right\|_{L^{p}}
$$

où l'on a posé

$$
\alpha(j, q)= \begin{cases}\frac{j-q}{|j-q|}, & \text { si } j \neq q, \\ 0, & \text { sinon. }\end{cases}
$$

Nous convenons que $\psi^{0}=\mathrm{Id}$.

Admettons pour le moment ce lemme et voyons comment il permet de conclure. $\mathrm{Vu}$ que le flot est une transformation qui préserve la mesure de Lebesgue, alors on peut écrire via le lemme 3 et les inégalités (17) et (6) que

$$
\begin{aligned}
\nu 2^{2 q}\left\|\omega_{q}\right\|_{L^{1}\left([0, t] ; L^{p}\right)}= & \nu 2^{2 q}\left\|\bar{\omega}_{q}\right\|_{L^{1}\left([0, t] ; L^{p}\right)} \\
\leq & \nu 2^{2 q} \sum_{j \geq q-N_{0}}\left\|\Delta_{j} \bar{\omega}_{q}\right\|_{L^{1}\left([0, t] ; L^{p}\right)}+ \\
& +\nu 2^{2 q} \sum_{j \leq q-N_{0}}\left\|\Delta_{j} \bar{\omega}_{q}\right\|_{L^{1}\left([0, t] ; L^{p}\right)} \\
\leq & C 2^{2 N_{0}} h(t)\left(\nu 2^{2 q}\right)\left\|\omega_{q}\right\|_{L^{1}\left([0, t] ; L^{p}\right)}+C 2^{3 N_{0}}\left\|\omega^{0}\right\|_{L^{p}} g(t)+ \\
+ & C\left\|\omega^{0}\right\|_{L^{p}}+C\left(\nu 2^{2 q}\right)\left\|\omega_{q}\right\|_{L^{1}\left([0, t] ; L^{p}\right)} 2^{-N_{0}} e^{C V(t)} .
\end{aligned}
$$


Sous cette forme on voit clairement le rôle que l'entier $N_{0}$ joue : dans la zone des hautes fréquences on profite de la petitesse (en temps) de la fonction $h$. Tandis que dans le spectre des basses fréquences on agrandit $N_{0}$ de manière à ce que le dernier terme figurant dans (18) soit absorbé par la quantité de gauche. Mais le choix du temps et de $N_{0}$ sont fortement liés. ils sont donnés par les deux conditions suivantes :

$$
C 2^{2 N_{0}} h(t) \leq \frac{1}{4} \quad \text { et } \quad C 2^{-N_{0}} e^{C V(t)} \leq \frac{1}{4}
$$

Par suite, si $V(t) \leq 1$, on choisit $N_{0}$ pour que $2^{-N_{0}} \leq \frac{1}{4 C} e^{-C}$, puis quitte à diminuer encore $V(t)$, on assure que $C 2^{2 N_{0}} h(t) \leq 1 / 4$. Ainsi on montre l'existence d'une constante $C_{0}$ telle que, si

$$
\int_{0}^{t}\|\nabla v(\tau)\|_{L^{\infty}} d \tau \leq C_{0}
$$

alors (19) aura lieu. Auquel cas l'entier $N_{0}$ est aussi absolu. Il s'ensuit que pour tout $t$ satisfaisant (20) et pour tout $q \geq N_{0}$,

$$
\left(\nu 2^{2 q}\right)^{\frac{1}{r}}\left\|\omega_{q}\right\|_{L^{r}\left([0, t] ; L^{p}\right)} \leq C\left\|\omega^{0}\right\|_{L^{p}}
$$

Pour finir cette première étape, il nous reste à voir comment s'estiment les basses fréquences : nous obtenons aisément, grâce à l'estimation

$$
\|\omega(t)\|_{L^{p}} \leq\left\|\omega^{0}\right\|_{L^{p}}
$$

et via la continuité uniforme en $q$ de l'opérateur de localisation en fréquence $\Delta_{q} \in \mathcal{L}\left(L^{p}\right)$, que

$$
\nu 2^{q}\left\|\Delta_{q} \omega\right\|_{L^{1}\left([0, t] ; L^{p}\right)} \leq C(\nu t)\left\|\omega^{0}\right\|_{L^{p}}
$$

Ainsi, nous parvenons à établir localement en temps, le résultat énoncé dans la proposition 2 .

\section{Estimation globale}

L'extension du résultat local obtenu à n'importe quel temps arbitraire positif $T$ n'est pas difficile. Nous parvenons à propager l'effet régularisant de proche en proche car la condition locale (20) ne tient pas compte des estimées de 
la solution $a$. Elle est uniquememt liée au gradient du champ de vecteurs $v$. Le point de départ de la preuve est de partager l'intervalle $[0, T]$ en une subdivision $\left(T_{i}\right)_{i=0}^{N}$ comme ci-dessous :

$T_{0}=0 \leq T_{1} \leq \ldots \leq T_{N}=T \quad$ et $\quad \int_{T_{i}}^{T_{i+1}}\|\nabla v(\tau)\|_{L^{\infty}} d \tau \simeq C_{0}, \forall i \in \llbracket 0, N-1 \rrbracket$.

Dans chacun de ces sous intervalles $\left[T_{i}, T_{i+1}\right]$, on reproduit exactement la même démarche locale sauf qu'au lieu d'utiliser le flot $\psi_{q}$ fixant l'espace à l'instant $t=0$, nous devons réinitialiser le flot à l'instant $T_{i}$. On obtient alors pour tout entier $q \geq N_{0}$ ( $N_{0}$ est le même entier qui apparaît dans la preuve locale)

$$
\begin{aligned}
\nu 2^{2 q}\left\|\omega_{q}\right\|_{L^{1}\left(\left[T_{i}, T_{i+1}\right] ; L^{p}\right)} & \leq C\left\|\omega\left(T_{i}\right)\right\|_{L^{p}} \\
& \leq C\left\|\omega^{0}\right\|_{L^{p}}
\end{aligned}
$$

Sachant que $N \simeq 1+\int_{0}^{T}\|\nabla v(\tau)\|_{L^{\infty}} d \tau$, alors en sommant les inégalités de 0 jusqu'au $N-1$, nous obtenons grâce à l'inégalité triangulaire que pour tout entier $q \geq N_{0}$

$$
\begin{aligned}
\nu 2^{2 q}\left\|\omega_{q}\right\|_{L^{1}\left([0, T] ; L^{p}\right)} & \leq \nu 2^{2 q} \sum_{i=0}^{N-1}\left\|\omega_{q}\right\|_{L^{1}\left(\left[T_{i}, T_{i+1}\right] ; L^{p}\right)} \\
& \leq C\left\|\omega^{0}\right\|_{L^{p}}\left(1+\int_{0}^{T}\|\nabla v(\tau)\|_{L^{\infty}} d \tau\right) .
\end{aligned}
$$

Ainsi l'estimation de la proposition 2 découle facilement des relations (22) et (24).

\section{Estimation Lipschitz de la vitesse}

Nous allons voir que l'estimation (1) combinée avec l'effet régularisant permettent de contrôler la quantité $\int_{0}^{t}\left\|\nabla v_{\nu}(\tau)\right\|_{L^{\infty}} d \tau$. Soit $N$ un entier positif. Alors l'inégalité triangulaire donne

$$
\begin{array}{r}
\int_{0}^{t}\left\|\nabla v_{\nu}(\tau)\right\|_{L^{\infty}} d \tau \leq \int_{0}^{t}\left\|\nabla S_{N} v(\tau)\right\|_{L^{\infty}} d \tau+\int_{0}^{t}\left\|\nabla S_{N}\left(v_{\nu}-v\right)(\tau)\right\|_{L^{\infty}} d \tau \\
+\int_{0}^{t}\left\|\nabla\left(\mathrm{Id}-S_{N}\right) v_{\nu}(\tau)\right\|_{L^{\infty}} d \tau
\end{array}
$$


Nous soulignons d'abord que l'inégalité de convolution entraîne

$$
\int_{0}^{t}\left\|\nabla S_{N} v(\tau)\right\|_{L^{\infty}} d \tau \leq \int_{0}^{t}\|\nabla v(\tau)\|_{L^{\infty}} d \tau
$$

D'un autre côté, nous avons par le biais de l'inégalité de Bernstein et l'estimation (1)

$$
\begin{aligned}
\int_{0}^{t}\left\|\nabla S_{N}\left(v_{\nu}-v\right)(\tau)\right\|_{L^{\infty}} d \tau & \leq C 2^{2 N} \int_{0}^{t}\left\|\left(v_{\nu}-v\right)(\tau)\right\|_{L^{2}} d \tau \\
& \leq C \nu t 2^{2 N} e^{\exp C t\left\|v^{0}\right\|_{B_{2,1}^{2}}}
\end{aligned}
$$

Pour la majoration du dernier terme de (25), on utilise la proposition 2, qui implique

$$
\begin{aligned}
\int_{0}^{t}\left\|\nabla\left(\mathrm{Id}-S_{N}\right) v_{\nu}(\tau)\right\|_{L^{\infty}} d \tau & \leq \sum_{q \geq N} \int_{0}^{t}\left\|\Delta_{q} \omega_{\nu}(\tau)\right\|_{L^{\infty}} d \tau \\
& \leq C \frac{\left\|\omega^{0}\right\|_{L^{\infty}}}{\nu 2^{2 N}}\left(1+\int_{0}^{t}\left\|\nabla v_{\nu}(\tau)\right\|_{L^{\infty}} d \tau\right)
\end{aligned}
$$

En reportant (26), (27) et (28) dans (25), on trouve

$$
\begin{aligned}
\int_{0}^{t}\left\|\nabla v_{\nu}(\tau)\right\|_{L^{\infty}} d \tau \leq C\left(1+\nu t 2^{2 N}\right) & e^{\exp C t\left\|v^{0}\right\|_{B_{2,1}^{2}}}+ \\
& +C \frac{\left\|\omega^{0}\right\|_{L^{\infty}}}{\nu 2^{2 N}}\left(1+\int_{0}^{t}\left\|\nabla v_{\nu}(\tau)\right\|_{L^{\infty}} d \tau\right)
\end{aligned}
$$

Par conséquent, en choisissant $N$ tel que

$$
C \frac{\left\|\omega^{0}\right\|_{L^{\infty}}}{\nu 2^{2 N}} \simeq \frac{1}{2}
$$

alors on aura

$$
\begin{aligned}
\int_{0}^{t}\left\|\nabla v_{\nu}(\tau)\right\|_{L^{\infty}} d \tau & \leq C\left(1+t\left\|\omega^{0}\right\|_{L^{\infty}}\right) e^{\exp C t\left\|v^{0}\right\|_{B_{2,1}^{2}}} \\
& \leq C e^{\exp C t\left\|v^{0}\right\|_{B_{2,1}^{2}}}
\end{aligned}
$$

Nous avons utilisé dans le dernier passage l'inclusion de Sobolev $(\mathrm{d}=2)$ :

$$
B_{2,1}^{1} \hookrightarrow L^{\infty} .
$$




\section{Estimation du commutateur}

Nous nous proposons dans cette section de fournir une estimation précise du commutateur $\left[\Delta_{q}, v \cdot \nabla\right] \omega$, où $\omega=\operatorname{rot} v$.

Proposition 3. Soit $v$ un champ de vecteurs régulier de $\mathbb{R}^{2}$, à divergence nulle. Soit $\omega$ son rotationnel, donné par $\omega=\partial_{1} v^{2}-\partial_{2} v^{1}$. Alors pour tout $q \geq-1$ et pour tout $p \in[1,+\infty]$ on a

$$
\left\|\left[\Delta_{q}, v \cdot \nabla\right] \omega\right\|_{L^{p}} \leq C\|\nabla v\|_{L^{\infty}} \sum_{j \geq q-N_{0}} 2^{q-j}\left\|\Delta_{j} \omega\right\|_{L^{p}}
$$

Les nombres $C$ et $N_{0}$ sont des constantes absolues.

Démonstration : Nous ferons usage de la décomposition de Bony [2]:

$$
\left[\Delta_{q}, v \cdot \nabla\right] \omega=\left[\Delta_{q}, T_{v} \cdot \nabla\right] \omega+\left[\Delta_{q}, T_{\nabla \cdot} \cdot v\right] \omega+\left[\Delta_{q}, R(v, \nabla \cdot)\right] \omega .
$$

L'estimation du second terme est facile. On écrit simplement

$$
\left\|\left[\Delta_{q}, T_{\nabla \cdot} \cdot v\right] \omega\right\|_{L^{p}} \leq C \sum_{|j-q| \leq N_{0}}\left\|S_{j-1} \nabla \omega\right\|_{L^{\infty}}\left\|\Delta_{j} v\right\|_{L^{p}}
$$

Or, nous avons par le lemme de Bernstein et par définition du tourbillon

$$
\begin{aligned}
\left\|S_{j-1} \nabla \omega\right\|_{L^{\infty}} & \leq C 2^{j}\|\omega\|_{L^{\infty}} \\
& \leq C 2^{j}\|\nabla v\|_{L^{\infty}} .
\end{aligned}
$$

D'un autre côté, la somme figurant dans (31) porte sur des entiers $j$ qui sont positifs, sinon l'opérateur $S_{j-1}$ est nul. Par conséquent, on aura par le biais du lemme de Bernstein,

$$
\left\|\Delta_{j} v\right\|_{L^{p}} \leq C 2^{-j}\left\|\Delta_{j} \omega\right\|_{L^{p}}
$$

Ainsi en reportant ces estimations dans (31), nous obtenons

$$
\left\|\left[\Delta_{q}, T_{\nabla \cdot} \cdot v\right] \omega\right\|_{L^{p}} \leq C\|\nabla v\|_{L^{\infty}} \sum_{|j-q| \leq N_{0}}\left\|\Delta_{j} \omega\right\|_{L^{p}}
$$

Concernant le second terme qui apparaît dans le second membre de 30, nous avons par définition du paraproduit et grâce à la commutation des opérateurs 
$\Delta_{q}$ entre eux

$$
\begin{aligned}
{\left[\Delta_{q}, T_{v} \cdot \nabla\right] \omega } & =\sum_{k=1}^{2} \sum_{j}\left[S_{j-1} v^{k} \partial_{k} \Delta_{j}, \Delta_{q}\right] \omega \\
& =\sum_{k=1}^{2} \sum_{j}\left[S_{j-1} v^{k} \partial_{k}, \Delta_{q}\right] \Delta_{j} \omega
\end{aligned}
$$

La somme est en fait finie et ne concerne que les indices $j$ vérifiant $|j-q| \leq$ $N_{0}$. Ceci est dû, d'une part, à la localisation du support de la transformée de Fourier de $S_{j-1} v^{k} \Delta_{j} \partial_{k} a$ dans une couronne de taille $2^{j}$ et d'autre part au fait que $\Delta_{q} \Delta_{j} \equiv 0$, si $|j-q| \geq 2$.

Pour majorer chacun de ces commutateurs, on écrit l'opérateur $\Delta_{q}$ comme une intégrale de convolution :

$$
\left[S_{j-1} v^{k} \partial_{k}, \Delta_{q}\right] \Delta_{j} \omega(\cdot)=2^{q d} \int h\left(2^{q}(\cdot-y)\right)\left(S_{j-1} v^{k}(\cdot)-S_{j-1} v^{k}(y)\right) \Delta_{j} \partial_{k} \omega(y) d y .
$$

Ainsi, l'inégalité de Young donne

$$
\begin{aligned}
\left\|\left[S_{j-1} v^{k} \partial_{k}, \Delta_{q}\right] \Delta_{j} \omega(\cdot)\right\|_{L^{p}} & \leq C 2^{-q}\left\|\nabla S_{j-1} v\right\|_{L^{\infty}}\left\|\Delta_{j} \partial_{k} \omega\right\|_{L^{p}} \\
& \leq C\|\nabla v\|_{L^{\infty}} 2^{j-q}\left\|\Delta_{j} \omega\right\|_{L^{p}} .
\end{aligned}
$$

En conséquence, nous aurons l'estimation

$$
\left\|\left[\Delta_{q}, T_{v} \cdot \nabla\right] \omega\right\|_{L^{p}} \leq C\|\nabla v\|_{L^{\infty}} \sum_{|j-q| \leq N_{0}} 2^{-|j-q|}\left\|\Delta_{j} \omega\right\|_{L^{p}}
$$

Le terme de reste s'écrit par définition comme:

$$
\left[\Delta_{q}, R(v, \nabla \cdot)\right] \omega=\sum_{\substack{j \geq-1 \\ i \in\{\mp 1,0\}}}\left[\Delta_{q}, \Delta_{j} v \cdot \nabla\right] \Delta_{j+i} \omega=\sum_{\substack{j \geq q-N_{0} \\ i \in\{\mp 1,0\}}}\left[\Delta_{q}, \Delta_{j} v \cdot \nabla\right] \Delta_{j+i} \omega .
$$

Il se décompose aussi de la manière suivante

$$
\begin{aligned}
& {\left[\Delta_{q}, R(v, \nabla \cdot)\right] \omega=R_{q}^{1}(v, a)+R_{q}^{2}(v, a), \text { avec }} \\
& R_{q}^{1}(v, \omega)=\sum_{\substack{q-N_{0} \leq j \leq q \\
i \in\{\mp 1,0\}}}\left[\Delta_{q}, \Delta_{j} v \cdot \nabla\right] \Delta_{j+i} \omega \text { et } \\
& R_{q}^{2}(v, \omega)=\sum_{\substack{q+1 \leq j \\
i \in\{\mp 1,0\}}}\left[\Delta_{q}, \Delta_{j} v \cdot \nabla\right] \Delta_{j+i} \omega .
\end{aligned}
$$


Pour estimer $R_{q}^{1}(v, \omega)$, on imite l'inégalité (33) et l'on trouve :

$$
\begin{aligned}
\left\|R_{q}^{1}(v, \omega)\right\|_{L^{p}} & \leq C \sum_{\substack{q-N_{0} \leq j \leq q \\
i \in\{\mp 1,0\}}} 2^{-q}\left\|\nabla \Delta_{j} v\right\|_{L^{\infty}}\left\|\nabla \Delta_{j+1} \omega\right\|_{L^{p}} \\
& \leq C\|\nabla v\|_{L^{\infty}} \sum_{\substack{q-N_{0} \leq j \leq q \\
i \in\{\mp 1,0\}}} 2^{j-q}\left\|\Delta_{j+i} \omega\right\|_{L^{p}} \\
& \leq C\|\nabla v\|_{L^{\infty}} \sum_{\substack{q-N_{0} \leq j \\
i \in\{\mp 1,0\}}} 2^{q-j}\left\|\Delta_{j} \omega\right\|_{L^{p}} .
\end{aligned}
$$

Par contre pour contrôler le terme $R_{q}^{2}(v, \omega)$ on utilise la condition de divergence nulle qui implique

$$
R_{q}^{2}(v, \omega)=\sum_{k=1}^{2} \sum_{\substack{q+1 \leq j \\ i \in\{\mp 1,0\}}} \partial_{k}\left[\Delta_{q}, \Delta_{j} v^{k}\right] \Delta_{j+i} \omega .
$$

En développant le commutateur figurant dans cette somme, on constate que sa transformée de Fourier est supportée dans une boule de taille $2^{q}$. Ainsi le lemme de Bernstein donne $(j \in \mathbb{N})$

$$
\begin{aligned}
\left\|\partial_{k}\left[\Delta_{q}, \Delta_{j} v^{k}\right] \Delta_{j+i} \omega\right\|_{L^{p}} & \leq C 2^{q}\left\|\Delta_{j} v^{k}\right\|_{L^{\infty}}\left\|\Delta_{j+i} \omega\right\|_{L^{p}} \\
& \leq C\|\nabla v\|_{L^{\infty}} 2^{q-j}\left\|\Delta_{j+i} \omega\right\|_{L^{p}} .
\end{aligned}
$$

Par suite, on aura

$$
\left\|R_{q}^{2}(v, \omega)\right\|_{L^{p}} \leq C\|\nabla v\|_{L^{\infty}} \sum_{q+1 \leq j} 2^{q-j}\left\|\Delta_{j} \omega\right\|_{L^{p}} .
$$

Ce qui achève la preuve de la proposition 3.

\section{Fin de la démonstration du théorème 1}

Pour prouver l'estimation de propagation dans $B_{2,1}^{2}$, il suffit de le faire pour le tourbillon dans l'espace $B_{2,1}^{1}$. On commence par appliquer l'opérateur de filtrage en fréquences $\Delta_{q}$ à l'équation qui régit le tourbillon visqueux et l'on trouve que $\omega_{q} \stackrel{\text { déf }}{=} \Delta_{q} \omega$ satisfait

$$
\partial_{t} \omega_{q}+v \cdot \nabla \omega_{q}-\nu \Delta \omega_{q}=-\left[\Delta_{q}, v \cdot \nabla\right] \omega .
$$


En prenant le produit scalaire $L^{2}$ avec $\omega_{q}$ et en se servant de l'incompressibilité du flot et de la proposition 3 , on trouve suite à une intégration en temps $\left\|\omega_{q}(t)\right\|_{L^{2}}^{2} \leq\left\|\omega_{q}(0)\right\|_{L^{2}}^{2}+2 \int_{0}^{t}\left\|\omega_{q}(\tau)\right\|_{L^{2}}\|\nabla v(\tau)\|_{L^{\infty}} \sum_{j \geq q-N_{0}} 2^{q-j}\left\|\Delta_{j} \omega(\tau)\right\|_{L^{2}} d \tau$.

En appliquant le lemme de Gronwall, on trouve

$$
\left\|\omega_{q}(t)\right\|_{L^{2}} \leq\left\|\omega_{q}(0)\right\|_{L^{2}}+2 \int_{0}^{t}\|\nabla v(\tau)\|_{L^{\infty}} \sum_{j \geq q-N_{0}} 2^{q-j}\left\|\Delta_{j} \omega(\tau)\right\|_{L^{2}} d \tau .
$$

Posons $c_{q}(t)=2^{q}\left\|\omega_{q}(t)\right\|_{L^{2}}$. Alors l'estimation ci-dessus peut s'écrire sous la forme

$$
c_{q}(t) \leq c_{q}(0)+2 \int_{0}^{t}\|\nabla v(\tau)\|_{L^{\infty}} \sum_{j \geq q-N_{0}} 2^{2(q-j)} c_{j}(\tau) d \tau .
$$

Il suffit à ce stade de sommer sur les $q$ et d'appliquer l'inégalité de Young pour aboutir à

$$
\|\omega(t)\|_{B_{2,1}^{1}} \leq\left\|\omega^{0}\right\|_{B_{2,1}^{1}}+\int_{0}^{t}\|\nabla v(\tau)\|_{L^{\infty}}\|\omega(\tau)\|_{B_{2,1}^{1}} d \tau
$$

On conclut par le lemme de Gronwall qui implique

$$
\|\omega(t)\|_{B_{2,1}^{1}} \leq\left\|\omega^{0}\right\|_{B_{2,1}^{1}} e^{\int_{0}^{t}\|\nabla v(\tau)\|_{L} \infty d \tau} .
$$

Pour estimer la vitesse dans $B_{2,1}^{2}$, on écrit

$$
\begin{aligned}
\|v(t)\|_{B_{2,1}^{2}} & \leq\left\|\Delta_{-1} v(t)\right\|_{L^{2}}+\sum_{q \in \mathbb{N}} 2^{2 q}\left\|\Delta_{q} v(t)\right\|_{L^{2}} \\
& \leq\|v(t)\|_{L^{2}}+\|\omega(t)\|_{B_{2,1}^{1}} .
\end{aligned}
$$

Or l'inégalité d'énergie nous assure que pour tout $t$ positif

$$
\|v(t)\|_{L^{2}} \leq\left\|v^{0}\right\|_{L^{2}} \leq\left\|v^{0}\right\|_{B_{2,1}^{2}} .
$$

Donc, en reportant cette estimation ainsi que (35) dans (36), on trouve

$$
\|v(t)\|_{B_{2,1}^{2}} \leq C\left\|v^{0}\right\|_{B_{2,1}^{2}} e^{\int_{0}^{t}\|\nabla v(\tau)\|_{L^{\infty} d \tau}}
$$

Il suffit maintenant d'utiliser le contrôle Lipschitz (29). 


\section{References}

[1] H. Bahouri et J.-Y. Chemin, Equations de transport relatives à des champs de vecteurs non-lipschitziens et mécanique des fluides, Arch. Rational Mech. Anal., 127, pages 159-181, 1994.

[2] J.-M. Bony, Calcul symbolique et propagation des singularités pour les équations aux dérivées partielles non linéaires, Annales de l'école supérieure, 14, pages 209-246, 1981.

[3] J.-Y. Chemin, Perfect incompressible Fluids, Oxford University Press.

[4] J.-Y. Chemin, A Remark on the inviscid limit for two-dimmensionnel incompressible fluid, Communications in Partial Differential Equations, 21, pages 1771-1779, 1996.

[5] P. Constantin and C. Foias, Navier-Stokes equations, Chicago Lectures in Mathematics, University of Chicago Press (1988).

[6] R. Danchin, The inviscid limit for density-dependent incompressible fluids.

[7] H. Fujita and T. Kato, On the nonstationnary Navier-Stokes system, rend. Sem. Mat. Univ. Padova, 32, pages 243 - 260, (1962).

[8] T. Hmidi, régularité höldérienne des poches de tourbillon visqueuses, Prépublication de L'Ecole Polytechnique, 2003.

[9] T. Kato and G. Ponce, Commutator estimates and the Euler and Navier-Stokes equations, Communications on Pure and Applied Mathematics, 41, pages $891-907$ (1988)

[10] A. Majda, Vorticity and the mathematical theory of an incompressible fluid flow, Communications on Pure and Applied Mathematics, 38, pages 187-220, $1986 .$.

[11] M. Vishik, Hydrodynamics in Besov Spaces, Arch. Rational Mech. Anal 145, pages 197-214, 1998. 